\title{
Desarrollo de la destreza de speaking en el idioma inglés mediante la técnica "Basic Context English" en estudiantes de 5 a 6 años
}

\author{
Development of speaking skill in the english language using the "Basic \\ Context English Technique " in students from 5 to 6 years old
}

Sandra Leticia Guijarro Paguay. ${ }^{1}$, María Veronica Pilco López. ${ }^{2}$, Mayra Alexandra Carrillo Rodríguez. ${ }^{3} \&$ Blanca Flor Santa Cruz Fajardo. ${ }^{4}$

\section{Recibido: 14-03-2020 / Revisado: 19-04-2020 /Aceptado: 05-05-2020/ Publicado: 05-06-2020}

\begin{abstract}
.
DOI: https://doi.org/10.33262/concienciadigital.v3i2.2.1248

The objective of this research was to develop the speaking skill in the English language through the use of "Basic Context English" technique in 30 students from 5 to 6 years old at "Luis Humberto Sancho" kindergarten of Riobamba city. For this purpose, an intervention plan was developed and implemented. It contains a set of dynamic recreational activities for students. They were guided by the teacher, considering the dimensions of articulation and pronunciation with basic vocabulary. The research was a quasi-experimental study, where the variables were analyzed without direct intervention while the data were obtained by the technique of observation. It was applied in two different moments, before and after the experimentation. For the evaluation, an instrument with a Likert scale was used. It made possible to quantify the obtained data so that it could be interpreted, analyzed and tabulated, and finally submitted to the T-Student test. It concluded that the articulation and pronunciation with basic vocabulary are linked to the learning of English in a playful manner allowing fluency, increase of vocabulary, and an appropriate pronunciation. It means it influenced in the development of speaking skill in the English language.
\end{abstract}

Keywords: Development of speaking skill, kindergarten, fluency, increase of vocabulary, appropriate pronunciation

\section{Resumen.}

El objetivo de la presente investigación fue desarrollar la destreza del speaking del idioma inglés mediante la utilización de la técnica "Basic Context English" en 30 estudiantes de 5 a

\footnotetext{
${ }^{1}$ Escuela Superior Politécnica de Chimborazo, Facultad de Mecánica, Riobamba, Ecuador, sandra.guijarro@espoch.edu.ec, https://orcid.org/0000-0002-0413-4925

2 Unidad Educativa del Milenio Penipe, Penipe, Ecuador, veronica.pilco@educacion.gob.ec https://orcid.org/0000-0001-9271-3937

${ }^{3}$ Escuela Superior Politécnica de Chimborazo, Centro de Idiomas, Riobamba, Ecuador, mayra.carrillo@espoch.edu.ec https://orcid.org/0000-0003-4162-7793

${ }^{4}$ Instituto Superior Tecnológico Riobamba, Centro de idiomas, Riobamba, Ecuador, blanqiscab19@gmail.com, https://orcid.org/0000-0003-0060-5985
} 
6 años del Jardín de Infantes "Luis Humberto Sancho" de la ciudad de Riobamba. Para ello, se elaboró y aplicó un plan de intervención que contiene un conjunto de actividades lúdicasdinámicas dirigidas a los estudiantes y guiadas por el docente, considerando las dimensiones de articulación y pronunciación con vocabulario básico. La investigación fue cuasi experimental, donde se analizó las variables sin intervención directa; mientras que los datos fueron obtenidos mediante la técnica de observación y fue aplicada en dos momentos antes y después de la experimentación. Para la evaluación, se utilizó un instrumento con escala de Likert, lo que permitió cuantificar los datos obtenidos para poderlos tabularlos, analizarlos, interpretados y someterlos a la prueba T-student, que permitió concluir que la articulación y pronunciación con vocabulario básico se vinculan de forma lúdica a los aprendizajes de inglés permitiendo fluidez, enriquecimiento de vocabulario y una pronunciación adecuada; es decir, que incidieron en el desarrollo de la destreza del speaking del idioma inglés.

Palabras Clave: Desarrollo, Destreza, Habla, Jardín, Fluidez, Vocabulario, Pronunciación

\section{Introducción.}

El sistema educativo en el Ecuador presenta graves deficiencias en la enseñanza del inglés, demostrándose esta afirmación, porque luego de diez niveles de escolarización obligatoria y tres más de bachillerato los estudiantes, no son capaces de comunicarse en otro idioma, ni siquiera de la manera más elemental, en este contexto, el presente artículo presenta los resultados de la evaluación de una técnica metodológica denominada "Basic Contex English" aplicada a niños de 5 y 6 años a través de actividades lúdicas en las que los estudiantes toman parte activa de su propio aprendizaje guiados por los docentes, la investigación completa comprende tres unidades funcionales de aprendizaje, sin embargo en este artículo se presenta el análisis de una de ellas, la adquisición del speaking a través de ejercicios de articulación y pronunciación con vocabulario básico (Guijarro, 2013).

La adquisición de una segunda lengua, sobre todo el idioma inglés, actualmente es una prioridad en el contexto del desarrollo adaptativo a los nuevos sistemas sociales y de producción. Para conseguirlo de forma rápida y eficiente los especialistas recomiendan iniciar con el aprendizaje de este idioma a tempranas edades (Cardona Serrano, Fandiño Parra, \& Bermudez, 2017; Castillo-Cedeño, Ramírez-Abrahams, \& Ruíz-Guevara, 2017), es importante recordar que los seres humanos aprenden, comprenden y hablan la lengua materna luego de un proceso de aprendizaje, en el que se escucha, se repite y se utiliza en sitiuaciones habituales con las personas con las que se tiene contacto en el entorno. Esta es la forma más efectiva e interesante de aprender otro idioma, de tal suerte que, muchos pedagogos de las lenguas extranjeras (Cardona Serrano et al., 2017) aconsejan a los docentes promover la adquisición natural en espacios de comunicación antes que la adquisición de vocabulario aislado, explicaciones de estructuras y adiestramientos gramáticales, mucho menos en niños pequeños. 
Según Bailey (2005) el proceso operativo se basa en una guia de actividades de aprendizaje por interacción, es decir la participación activa de los estudiantes con el propósito de confirmar que "...una buena enseñanza no es la que muestra a los estudiantes lo que el docente sabe, sino cuanto los ayuda a mejorar sus conocimientos, destrezas y desempeño". (p.59)

Se utilizó una técnica a la que se denominó "Basic Context English" que consiste en un proceso de escuchar lo que pronuncia el docente, mirar mímicas, acciones o gráficos posterior relacionarlo con lo escuchado y observado, repetir palabras o frases en voz alta a fin de adiestrar el oido, familiarizarse con el significado y reflejarlo en lo aprendido dentro de un contexto dado.

\section{EI Procesos del aprendizaje.}

Para Gagné (2012), los procesos de aprendizaje son el resultado de las interacciones entre las personas y el ambiente, produciendo cambios de tipo comportamental, conductual e incluso de disposición y actitud respecto a una parte de la totalidad de la realidad, que se han mantenido en el transcurso del tiempo, no solo por el desarrollo madurativo, sino por las vivencia de experiencias. Explica que la información ingresa al sistema nervioso central a través de los receptores sensoriales, para luego ser procesada y almacenada en la memoria, hasta cuando se requiera, y se dé el proceso de recuperación. Si la información tiene correspondencia con alguna otra anterior, su almacenamiento resulta sencillo, pero si no es así, es necesaria la asimilación a través de la práctica, la repetición y la fijación de los aprendizajes. Las emociones intensas y las motivaciones contribuyen a facilitar o bloquear el almacenamiento de la información y su posterior recuperación según sea el caso.

Núñez \& Silver (2002) sostiene que el propósito del aprendizaje se orienta principalmente en formar al individuo con la capacidad de determinar problemas de su contexto para analizarlas y reaccionar sobre las mismas con el fin de proponer una solución; por tanto, el propósito eficiente de aprender es aportar de forma positiva y eficaz ante la sociedad de forma participativa, donde el individuo pueda analizar su entorno en su contexto, desarrollar ideas, y resolver los problemas que se presenten en su vida social.

En la investigación de Guijarro (2013) cita a Márquez y La Salle (2012) La información y los estímulos del ambiente, se receptan a través de los receptores, que son estructuras en el sistema nervioso central del individuo. De allí pasan al llamado registro sensorial, que es una estructura hipotética que fija objetos y los eventos que deben ser codificados de tal forma que obtienen validez para el cerebro. Esta información pasa a la memoria de corto plazo donde es nuevamente codificada, pero esta vez de forma conceptual.

Así mismo Guijarro (2013) referencia a Gagné (2012) para indicar que el autor considera que una vez que la información haya sido registrada y operada por cualquiera de las dos memorias, no presenta estructuras diferentes sino momentos, los mismos que puede ser 
eliminados o recuperada sobre la base de estímulos externos que hagan necesaria esa información.

Guijarro (2013) cita a varios autores sobre la teoría de procesos de aprendizaje; entre ellos a Gagné (2013) con su teoría donde propone y coincide en varios aspectos con el conductismo y el constructivismo. Teorías a las que integró aspectos de su propia investigación, como se menciona anteriormente, permite vincular los estímulos y al mismo tiempo propone que las fases del aprendizaje deben constituirse en un todo operativo si se quiere alcanzar resultados. En este sentido el propósito del pedagogo fue el de construir una estructura que se constituyera en la base que sustente la teoría de la instrucción. Así, a partir de los preceptos cognitivistas va incorporando aspectos del aprendizaje cognitivo y de los contenidos conductistas mantienen los criterios de los refuerzos y la evaluación de las tareas propuesto por (Skinner, 1978) (Ortega \& Romero, 2004). Para completar su enfoque se sirve de las teorías sobre el procesamiento de la información que le permiten alcanzar el esquema explicativo básico de las condiciones internas (Mergel, 1998).

Para fijar los conocimientos se aplica la fase de retención, en la cual los aprendizajes se integran a la memoria de corto plazo, se aumenta la información y se vincula nueva información dentro de las estructuras preestablecidas. De esta manera los contenidos se complementan y se acumulan permitiendo el ingreso a la fase de recuerdo, para en lo posterior poder activar la memoria a largo plazo, esto se logra generalmente a través del uso de estrategias que los docentes aplican para ayudar al estudiante a recordar el aprendizaje adquirido.

Finalmente, se impulsa la fase de generalización, en la cual el objetivo principal es la trasferencia global y la aplicación de los aprendizajes en situaciones diferentes. Esto da lugar a las dos últimas fases de los procesos de aprendizaje que son: la ejecución, en la que se puede observar el cambio de comportamiento del estudiante y la retroalimentación en la que se le propone al alumno un feedback como recopilación de todo el proceso.

La importancia de esta teoría a nivel educativo es relevante, permite la comprensión de los principios que rigen los procesos de aprendizaje, fortalecer la certeza de que el aprendiz este consiente de ellos y de que tiene el control de su propio aprendizaje, esto es el aprender a aprender(Galvis, 1997).

\section{Recursos Didácticos}

Uno de los elementos que posibilitan el desarrollo de los procesos de aprendizaje son los recursos didácticos, insustituibles en los primeros años de enseñanza básica y fundamentales en los posteriores periodos de educación, por esta razón es importante hacer una breve referencia sobre ellos vinculándolos al contenido de este artículo desde la perspectiva de la enseñanza de lenguas extranjeras.

Guijarro (2013) indica que los recursos y materiales didácticos son todo el conjunto de elementos, útiles o estrategias que el profesor utiliza, o puede utilizar, como soporte, complemento o ayuda en su tarea docente; por lo tanto, deben considerarse como un apoyo en el proceso educativo. 
El recurso y materiales didácticos, en educación tiene dos acepciones distintas, de manera general se considera la edificación, mobiliario, audiovisual, bibliografía, etc. Desde el punto de vista metodológico, a los recursos son concebidos como estrategias que son usadas por el profesor para facilitar la tarea docente, tanto en los aspectos organizativos como a también en la manera de transferir los conocimientos o contenidos.

Si bien, los recursos y materiales didácticos no son los elementos más importantes en la educación escolar, pues el papel primordial corresponde al elemento humano (profesor y alumno), algunos de ellos resultan imprescindibles para poder realizar la práctica educativa, pues se enmarcan en la función de mediar la intencionalidad educativa con el proceso de aprendizaje, concebido entre: el quien educa y el educando. La función mediadora general se deriva en diversas funciones específicas con el fin de cumplir con los recursos del proceso formativo; entre ellas:

- Estructuradora de la realidad,

- Estructura motivadora,

- Control de los contenidos de aprendizaje,

- Innovación, etc.

De los ítems antes mencionados, los recursos eximen funciones de influencia en el proceso educativo.

Fullan, que es citado por (Marchesi, 2008), menciona que "cualquier innovación inevitablemente contemplará el uso de materiales curriculares distintos a los utilizados habitualmente".

Desde la experiencia docente, los recursos materiales y didácticos deben cumplir con funciones básicas orientados al soporte de contenidos curriculares para convertirse en elementos que permitan realizar las actividades de enseñanza-aprendizaje.

Escudero (2003), propone funciones para los recursos didácticos: entre ellas las función motivadora: con el fin de captar la atención de los alumnos a través de un poder de atracción acreditado por formas, colores, tacto, acciones, sensaciones, etc. Además, agrega la función estructuradora, que se deben asumir constituyendo los medios entre la realidad y el conocimiento, con objetivo de cumplir funciones de organización de aprendizajes y brindar una alternativa a la misma realidad. Propone también la función didáctica de estricto e imprescindible congruencia entre recursos materiales, objetivos y contenidos objeto de enseñanza. Finalmente, la función facilitadora de aprendizaje de un idioma, que no serían alcanzados sin la existencia de ciertos recursos y materiales.

Guijarro (2013) en su investigación cita a . Kelly, (2000) para indicar que los profesores necesitan considerar como los sonidos utilizados son receptados y además se necesita estudiar su fisiología asegurando que:

Todos utilizamos los mismos órganos del habla para producir los sonidos que estamos acostumbrados a producir. El grupo de sonidos que adquirimos, sin embargo, puede variar: un niño educado en un ambiente de inglés desarrollará los 
fonemas del inglés, un niño que hable francés desarrollará un conjunto diferente, y así sucesivamente.

También se aprende a utilizar las voces de diferentes maneras: entre ellas como el niño hablante de inglés debe aprender a utilizar la acentuación, así como los modelos de entonación apropiados. El niño hablante de cantones deberá a aprender a utilizar los tonos para distintos significados del mismo conjunto de sonidos.

Para otras situaciones se debe aprender a usar los órganos del habla de tal manera de producir diferentes sonidos aprendidos de un idioma extranjero, o perder los sonidos del lenguaje materno que no son apropiados en un idioma extranjero. Sin embargo, después de la niñez la habilidad para adoptar un grupo de sonidos no familiar disminuye por su condición y adaptación aprendidos.

Los niños desarrollan la habilidad de producir sonidos del habla a diferentes edades. Por ejemplo, las investigaciones muestran que los niños de dos años de edad son entre 50 y $75 \%$ comprensibles, mientras que los niños de tres años son entre 75 a $100 \%$ comprensibles(Kelly, 2000; Navarro Romero, 2019). Es decir que es normal, que un niño de 3 años hable, y que el adulto solo entiende tres cuartas partes de lo que habla o menciona, o que un adulto logre comprender lo que un niño de 3 años pueda hablar. Además, los niños desarrollan las habilidades de articulación a menor ritmo que las niñas. Ambos ejemplos son considerados normales.

En base a la investigación se puede indicar que los niños crecen, aprenden a utilizar progresivamente los sonidos del lenguaje que pudieron ser difíciles de pronunciar en el pasado (Kelly , 2001). La siguiente lista incluye las edades aproximadas en las cuales los niños normalmente desarrollan la habilidad de producir correctamente sonidos específicos:

Tabla 1. Desarrollo de la articulación

\begin{tabular}{ll}
\hline \multicolumn{1}{c}{ Sonidos del lenguaje } & \multicolumn{1}{c}{$\begin{array}{c}\text { Edades en las cuales } \\
\text { normalmente los sonidos se } \\
\text { desarrollan }\end{array}$} \\
\hline $\mathrm{p}, \mathrm{m}, \mathrm{h}, \mathrm{w}$ & De 12 meses a 3 años \\
$\mathrm{B}$ & De 18 meses a 3 años y $1 / 2$ \\
$\mathrm{t}, \mathrm{d}$ & De 2 a 3 años $1 / 2$ \\
$\mathrm{k}, \mathrm{g}$ & De 2 a 4 años \\
$\mathrm{n}, \mathrm{ng}$ & De 2 años $1 / 2$ a 5 años \\
$\mathrm{f}, \mathrm{y}$ & De 3 años $1 / 2$ a $51 / 2$ \\
$\mathrm{~L}$ & De $2 \frac{1}{2}$ a 6 años $(7$ años para los \\
$\mathrm{R}$ & chicos) \\
$\mathrm{s}, \mathrm{z}$ & De $2 \frac{1 / 2}{2}$ a 8 años \\
$\mathrm{sh}$, & De 3 a 9 años \\
& De 4 a 6 años $(7$ años para los \\
chicos)
\end{tabular}




$\begin{array}{ll}\text { ch,j } & \begin{array}{l}\text { De } 3 \frac{1}{2} \text { a } 6 \text { años (7 años para } \\ \text { chicos) }\end{array} \\ \text { V } & \text { De } 4 \text { to } 5 \frac{1}{2} \text { años } \\ \text { No sonoro th (como en "thumb" } & \text { De } 4 \frac{1}{2} \text { a } 8 \text { años } \\ \text { Sonoro th (como en "that") } & \text { De } 5 \text { a } 7 \text { años } \\ \text { Combinación-s : sp, st, sk } & \text { De } 3 \text { a } 6 \text { años } \\ \text { Combinación }-\mathrm{s}: \text { sm, sn } & \text { De } 3 \frac{1}{2} \text { a } 7 \text { años } \\ \text { Combinación }-1 & \text { De } 4 \text { a } 6 \text { años } \\ \text { Combinación }-\mathrm{r} & \text { De } 4 \frac{1}{2} \text { a } 6 \text { años }\end{array}$

Nota: Las edades se aplican para inglés como primera lengua.

Los aprendices del inglés como segunda lengua con frecuencia desarrollan los sonidos a edades más tardías.

Fuente: (Maybloom, 2016)

Elaborado por: Sandra Guijarro

\section{Metodología.}

La Investigación fue de carácter cuasi experimental, que analiza variables observadas sin intervención directa, donde los resultados fueron sistematizadas tal y como se presentaron para ser contrastados, en un análisis donde se trabajó en dos escenarios antes y después de la aplicación de la técnica "Basic Context English", considerando las relaciones causales que determinan el desarrollo del aprendizaje del idioma inglés y el contexto en el que aprenden los niños.

La técnica utilizada fue la observación, que valoró el nivel de desarrollo de la destreza del speaking del idioma inglés; mientras que el instrumento fue una ficha de observación y evaluación que posibilitaron hacer una comparación del antes y el después de la aplicación del plan de intervención. La muestra fue intencional y está compuesta por un grupo de 30 niños con edades comprendidos entre las edades de 5 y 6 años pertenecientes a un paralelo del Jardín de Infantes "Luis Humberto Sancho" de la ciudad de Riobamba.

Inicialmente se aplicó una evaluación de diagnóstico, que demostró que a pesar de utilizar un lenguaje claro e instrucciones sencillas los estudiantes poseían un escaso o casi nulo vocabulario básico para ese nivel, además que muchos no podían pronunciar las palabras o frases de manera repetitiva y peor aún decirlas de manera espontánea frente a una situación específica.

En base a los resultados obtenidos, se elaboró y aplicó una intervención utilizando la Técnica Basic Context English, diseñada para el desarrollo de la destreza de speaking a través de tres estrategias básicas y dos complementarias. Las tres estrategias básicas son:

1. Ejercicios de pre-articulación que fueron diseñados para fortalecer los diferentes órganos del habla entre ellos: la boca, lengua, quijada y respiración, pues el saber 
inhalar y exhalar el aire es parte fundamental dentro de la correcta pronunciación de sonidos. Además, 7 ejercicios de articulación que se deben desarrollar uno a uno por medio de pasos sencillos con vocabulario específico para el primer año de educación general básica.

2. Canciones y rimas que fueron concebidas para la ayudar a los estudiantes a pronunciar las palabras mediante el correcto uso del acento y frases, oraciones y preguntas con un ritmo de pronunciación aceptable y comprensible para otra persona.

3. Juegos didácticos que fueron diseñados para que el estudiante desarrolle o produzca el lenguaje de los contenidos básicos establecidos en el último Currículo Nacional de inglés mediante competencias para niños de 1ro a $7 \mathrm{mo}$ año de Educación General Básica del año 2007.

4. Dos estrategias complementarias fueron: siete hojas de refuerzo, distribuidas en una para cada unidad, las mismas que contienen actividades de speaking y motrices que reforzaron lo aprendido.

5. Los modelos del material didáctico fueron sugeridos como complemento a la aplicación de la técnica. A fin de lograr eficiencia en la aplicación de la técnica en las actividades del aula de clase; los ejercicios de pre y articulación, canciones y rimas y juegos didácticos compuestos por un conjunto de pasos a seguir para cada actividad y la sugerencia del material didáctico a utilizar.

Los resultados obtenidos de la primera parte de la aplicación de la técnica "Basic Context English" en el que se comparan las capacidades de la utilización de los órganos que intervienen en el proceso de fonación que se constituye en parte primordial en el contexto del logro de una correcta pronunciación con la aplicación de ejercicios para la adquisición de vocabulario específico para el nivel. En base a lo cual se realizó el siguiente planteamiento hipotético. La aplicación de la Técnica "Basic Context English" desarrolla la destreza del speaking del idioma inglés a través de ejercicios de articulación y pronunciación con vocabulario básico en los estudiantes del Primer Año de Educación Básica del Jardín "Luis Humberto Sancho".

El instrumento para la recolección de los datos utilizó fue una ficha de cotejo mediante una escala de linkert que permitió cuantificar las dimensiones para ponderarlos. Los datos obtenidos de las evaluaciones diagnósticas y luego de aplicar el plan de intervención fueron filtradas, para posterior ser tabuladas, analizadas e interpretadas. Para compararlas mediante una prueba estadística T-student la misma que ayudó a la comprobación de la hipótesis planteada en el contexto de la presente investigación.

\section{Resultados}

Los resultados obtenidos de las evaluaciones realizadas antes y después de la aplicación de la técnica para el desarrollo del Speaking "Basic Context English", relacionadas con el aprendizaje de la pronunciación a través del mejoramiento de la articulación fonológica, se pueden observar en la Tabla 2. 
Tabla 2. Tabla comparativa de la evaluación de articulación, antes y después de la aplicación de la Técnica "Basic Complet English"

\begin{tabular}{|c|c|c|c|c|c|c|c|c|c|}
\hline \multirow[t]{2}{*}{ INDICADOR } & \multicolumn{2}{|c|}{$\begin{array}{c}\text { Sonidos Plosivos } \\
\text { /T/; /G/. }\end{array}$} & \multicolumn{2}{|c|}{$\begin{array}{c}\text { Sonidos } \\
\text { Fricativos } \\
\text { /F/; /S/; /H/ }\end{array}$} & \multicolumn{2}{|c|}{$\begin{array}{c}\text { Sonido } \\
\text { Aproximado } \\
\text { /R/ }\end{array}$} & \multicolumn{2}{|c|}{$\begin{array}{c}\text { Sonido Vocal } \\
\qquad / \mathbb{E} /\end{array}$} & \multirow[t]{2}{*}{ Total } \\
\hline & Antes & Después & Antes & Después & Antes & Después & Antes & Después & \\
\hline Muy Satisfactorio & 0 & 10 & 2 & 18 & 0 & 13 & 0 & 9 & 52 \\
\hline Satisfactorio & 28 & 20 & 22 & 12 & 23 & 17 & 8 & 19 & 149 \\
\hline $\begin{array}{l}\text { Medianamente } \\
\text { Satisfactorio }\end{array}$ & 2 & 0 & 6 & 0 & 7 & 0 & 22 & 2 & 39 \\
\hline Total & 30 & 30 & 30 & 30 & 30 & 30 & 30 & 30 & 240 \\
\hline
\end{tabular}

Fuente: Estadística Inicial y final

Elaborado por: Sandra Guijarro

Los resultados de las evaluaciones permiten establecer que los indicadores de satisfacción varían en función del grado de dificultad de la pronunciación de los sonidos, ya sea antes o después de la aplicación de la técnica; sin embargo, las diferencias en el aumento de las competencias de pronunciación han aumentado considerablemente, al compararse las dos evaluaciones. Sin embargo, la mayor cantidad de estudiantes se encuentran en los niveles de satisfactorio, reconociéndose que, para la evaluación posterior a la aplicación de la técnica de aprendizaje el número de estudiantes que alcanzaron calificaciones de muy satisfactorio, es muy inferior al que alcanzaron los alumnos en la evaluación inicial.

Se buscó establecer que la diferencia entre los dos momentos establecidos para la evaluación es significativa, por lo tanto, se realizó un resumen de los datos y se utilizó una prueba de Tstudent para muestras relacionadas encontrándose lo siguiente:

Tabla 3. Estadísticos de muestras relacionadas

\begin{tabular}{llrrrr}
\hline \multirow{2}{*}{ Par 1 } & & Media & N & Desviación típ. & Error típ. de la media \\
\cline { 3 - 6 } & Después & 6,93 & 120 & 1,086 &, 099 \\
& Des & 8,28 & 120 &, 972 &, 089 \\
\hline
\end{tabular}

Fuente: Estadística Inicial y final

Elaborado por: Sandra Guijarro

Del análisis de los datos, se estableció una media de calificación de 6,93 para 120 casos, considerando los cuatro tipos de sonidos fonéticos y los resultados de la evaluación en base a los tres indicadores propuestos, con los mismos parámetros la evaluación realizada después de la aplicación de la Técnica "Basic Context English" indicó una media de 8,28, estableciéndose que el promedio de calificación de la evaluación subió en 1,35 puntos. 
A continuación, se determina que la diferencia de puntaje es significativa, que permite concluir que la técnica "Basic Context English", que vinculada con la pronunciación ha generado un cambio significativo en el aprendizaje del idioma ingles en los niños de primer año de Educación Básica del jardín de Infantes Luis Humberto Sancho, para el efecto se aplicó la prueba de $\mathrm{T}$ de student para muestras relacionadas, obteniéndose los siguientes resultados Tabla 4.

Tabla 4. Prueba de $\mathbf{T}$ de student para muestras relacionadas

\begin{tabular}{|c|c|c|c|c|c|c|c|c|c|}
\hline & & \multicolumn{5}{|c|}{ Diferencias relacionadas } & \multirow[t]{3}{*}{$\mathrm{t}$} & \multirow[t]{3}{*}{$\mathrm{gl}$} & \multirow{3}{*}{$\begin{array}{c}\text { Sig. } \\
\text { (bilateral) }\end{array}$} \\
\hline & & \multirow[t]{2}{*}{ Media } & \multirow[t]{2}{*}{$\begin{array}{l}\text { Desviación } \\
\text { típ. }\end{array}$} & \multirow[t]{2}{*}{$\begin{array}{l}\text { Error típ. } \\
\text { de la } \\
\text { media }\end{array}$} & \multicolumn{2}{|c|}{$\begin{array}{c}95 \% \text { Intervalo de } \\
\text { confianza para la } \\
\text { diferencia }\end{array}$} & & & \\
\hline & & & & & Inferior & Superior & & & \\
\hline $\begin{array}{l}\text { Par } \\
1\end{array}$ & $\begin{array}{l}\text { ANTES - } \\
\text { DESPUES }\end{array}$ & $-1,358$ & 499 & ,046 & $-1,448$ & $-1,268$ & $-29,839$ & 119 &, 000 \\
\hline
\end{tabular}

Fuente: Estadística Inicial y final

Elaborado por: Sandra Guijarro

Donde con una media de 1,358 y una desviación típica de 0,499 , con 119 grados de libertad se obtiene un valor de T de $-29,839$ y una significancia bilateral de 0,000 , lo que indica que se acepta la hipótesis propuesta por el investigador afirmando que la aplicación de la Técnica "Basic Context English" desarrolla la destreza de speaking del idioma inglés a través de ejercicios de articulación y pronunciación con vocabulario básico en los estudiantes del Primer Año de Educación Básica del Jardín "Luis Humberto Sancho".

\section{Discusión.}

La creencia, que los niños son capaces de aprender otros idiomas con mayor facilidad que los adultos, es un supuesto muy extendido y sobre el cual incluso se han realizado investigaciones, así un estudio realizado en España relacionado a la opinión de los padres sobre el aprendizaje del inglés de sus hijos, el 92\% aseguro que estaban convencidos de las ventajas de los niños sobre los adultos en el aprendizaje de una segunda lengua (Torres, Traganl, \& García, 1997).

Este punto de vista, que subyace en la posibilidad de la existencia de un proceso evolutivo en la adquisición del lenguaje que duraría hasta el inicio de la pubertad y que sería comparable con el de la adquisición del lenguaje nativo, parte del hecho de que para los niños la adquisición del lenguaje se presenta de forma espontánea, por el contrario en el adulto ocurre una enorme variabilidad de situaciones que frenan el desarrollo de las competencias para lograr hablar una segunda lengua (L2), siendo muy difícil emular el nivel que se ha adquirido en el idioma nativo (Cardona Serrano et al., 2017) 
Al margen de las investigaciones, en las que se ha reportado la existencia de diferencias debidas a la edad es fundamentalmente en los niveles de producción de los aprendices de una segunda lengua considerando el contexto natural lo que ha servido para apoyar esta teoría que formula la denominada hipótesis del periodo crítico: en la que se afirma que los aprendices de menor edad tienen mayor éxito en el aprendizaje, la pubertad señala el declive en las capacidades de adquisición de una segunda lengua(Navarro Romero, 2019)

Por tanto se evidencia que los niños tienen mejores condiciones de aprendizaje para adquirir una segunda lengua, antes que los adultos, por lo que mientras más rápido comiencen los procesos de adquisición, los resultados obtenidos serán superiores. Sin embargo resulta importante establecer el momento oportuno para evitar interferencias el aprendizaje de la lengua materna, Klen (1986) sugiere que se debe iniciar la enseñanza de la segunda lengua cuando el niño ya domine la lengua materna y generalmente esto suele ocurrir entre los 3 y 4 años de edad. En los casos en los que el niño se desarrolle en un ambiente en el que desde su nacimiento se hablen dos lenguas, desarrollara paralelamente los dos sistemas gramaticales, en este sentido se está hablando del bilingüismo (Fleta, 2006).

Una vez que se ha establecido la pertinencia de iniciar la enseñanza del inglés lo antes posible es importante establecer cuáles son los aportes en cuanto al desarrollo de estrategias para integrar una adecuada pronunciación en el proceso de enseñanza aprendizaje, al respecto De la A Salinas (2016), asegura en su estudio realizado sobre estrategias para enseñar la pronunciación del inglés a niños de segundo año de Educación General Básica, que el 89\% de los padres de familia están a favor de una práctica permanente del idioma, sin embargo de ellos solamente el 59\% está de acuerdo en que se considere la pronunciación como una prioridad por lo que la autora considera que las estrategias más adecuadas para este propósito son las canciones utilizando técnicas de socialización y afectividad fomentando la inter actividad.

Por su parte Alcedo y Chacón (2011), consideran que el aprendizaje de la pronunciación de la segunda lengua en niños de edad escolar se facilita significativamente a través de actividades lúdicas, en el que se integre juegos psicomotores con el lenguaje, en base a estudios comparados muestran el impacto favorable de las actividades lúdicas como enfoque pedagógico funcional y motivacional en el aprendizaje del inglés, insisten además en que el docente que se especializa en esta área considere el desarrollo evolutivo del niño y la teorías psicolingüísticas que son fundamento de los procesos cognitivo y afectivo y que se considere la enseñanza de forma holística y en función de las necesidades biopsicosociales del niño en cada etapa.

Se han considerado dos elementos para esta discusión; en primer término, se establece contundentemente la ventaja que el niño tiene sobre el adulto; mientras que el segundo aspecto hace referencia a las necesidades psicoactivas y lúdicas que implica el aprendizaje de la segunda lengua en niños en educación inicial, aspectos que como se ha visto coinciden y están relacionados con la técnica "Basic Context English", que utiliza elementos lúdicos 
en un proceso de escucha y mímica dirigidas, la presentación de gráficos y la utilización de situaciones o acciones, que hacen posible la repetición de las palabras en voz alta, adiestrando de esta manera al oído y familiarizándole con el significado y la pronunciación en un contexto dado (Guijarro Paguay, 2017).

\section{Conclusiones.}

- La aplicación de la Técnica "Basic Context English, ha demostrado ser una herramienta didáctica eficiente para la adquisición de la destreza de speaking en niños de entre 5 y 6 años de edad, los procedimientos empleados por el docente utilizando articulación y pronunciación con vocabulario básico se vinculan de forma lúdica a los aprendizajes de inglés permitiendo fluidez, enriquecimiento de vocabulario y una pronunciación adecuada, haciendo posible que el desarrollo de conocimientos posteriores resulten más sencillos y permitiendo que el estudiante alcance logros significativos en la adquisición de otro idioma en los niveles superiores de escolarización.

- Se ha establecido a través de este trabajo de investigación que la adquisición de una lengua extranjera es más eficiente cuando se inicia en niños de entre 3 a 5 años, las características fisiológicas auditivas y motrices y el desarrollo de las capacidades fonéticas han alcanzado en este punto la madurez necesaria como para que el niño sea capaz de interiorizar el lenguaje sin la interferencia de la lengua materna y logre un speaking adecuado, como base funcional para continuar en su proceso de adquisición de una segunda lengua en la integración de las otras destrezas complementarias como las de leer y escribir.

\section{Referencias Bibliográficas.}

Alcedo , Y., \& Chacón, C. (2011). El enfoque lúdico como estrategia metodológica para promover el aprendizaje del inglés en niños de educación primaria. Saber. Revista Multidisciplibnaria del Consejo de investigación de la Universidad de Oriente, 23(1), $69-76$.

Bailey, K. M. (2005). Practical English Language Teaching: Speaking. McGraw-Hill ESL/ELT. https://doi.org/10.1021/acs.joc.6b00331

Cardona Serrano, A., Fandiño Parra, Y., \& Bermudez, J. (2017). La formación del profesorado de lengua extranjera como factor decisivo para la excelencia en la educación bilingüe. Ruta Maestra.

Castillo-Cedeño, R., Ramírez-Abrahams, P., \& Ruíz-Guevara, L. S. (2017). Necesidades de formación profesional en el ámbito de la primera infancia: Percepción y aportes del estudiantado. Revista Electronica Educare. https://doi.org/10.15359/ree.21-1.9

De La A Salinas , L. (2016). Learning strategies to develop english pronunciation for second basic grade students at. Santa Elena: Universidad Estatal de la peninsula de Santa Elena

Díaz Lucea, J. (1996). Los recursos y materiales didácticos en Educación Física. Apunts: Educación física y deportes.

Escudero Muñoz, J. M. (2003). La calidad de la educación: grandes lemas y serios 
ISSN: 2600-5859

interrogantes - Dialnet. dialnet. Recuperado de https://dialnet.unirioja.es/servlet/articulo? codigo $=2973312$

Fleta, T. (2006). Aprendizaje y técnicas de enseñanza del inglés en la escuela . Encuentro: Revista de investigación e innovación en la clase de lenguas, 51-62.

Gagné, R. (2012). Las condiciones del aprendizaje. Barcelona: McGraw - Hill

Galvis, A. H. (1997). Micromundos Lúdicos Interactivos: Aspectos Críticos en su Diseño y Desarrollo. Recuperado de http://zeus.uniandes.edu.co/

Guijarro Paguay, S. L. (2017). Creación y aplicación de la técnica "Basic Context English" para desarrollar la destreza del Speaking del idioma inglés en los estudiantes del primer año de Educación Básica del jardín Luis Humberto Sancho" de Riobamba durante el período septiembre 2013. Riobamba: UNACH.

Kelly, G. (2000). How to teach pronunciation. How to. https://doi.org/10.1017/CBO9781107415324.004

Klen, W. (1986). Second Language Acquisition . Canbridge: University Press

Marchesi, Á. (2008). Las emociones y los valores del profesorado. Ábaco.

Marquez, S., \& La Salle, A. (2012). Genomasur. Obtenido de El sistema Nervioso: http://www.genomasur.com/BCH/BCH_libro/capitulo_09.htm

Mergel, B. (1998). Diseño Instruccional Y Teoría Del Aprendizaje.

Navarro Romero, B. (2019). Adquisición De La Primera Y Segunda Lengua En Aprendientes En Edad Infantil Y Adulta. PhilUr, 2, 14 (115-128). Recuperado de https://w3.ual.es/revistas/PhilUr/pdf/PhilUr2.2010.Navarro.pdf

Núñez, C., Elena, M., \& Silver, A. (2002). Guía para la elaboración de materiales educativos orientados al aprendizaje autogestivo. Universidad de Guadalajara, ....

Ortega, J. B. F., \& Romero, E. Q. (2004). Los dos principios irrenunciables del análisis funcional de la conducta y del conductismo radical, 16(4), 555-562.

Torres, R., Traganl, E., \& García, M. (1997). Creencias populares sobre el aprendizaje temprano. AILE(10), 127-158.

Skinner, B. F. (1978). Conductismo Y Sociedad. Trillas. 


\section{PARA CITAR EL ARTÍCULO INDEXADO.}

Guijarro Paguay, S. L., Mantilla Cabrera, L. F., Carrillo Rodríguez, M. A., \& Pilco López, M. V. (2020). Desarrollo de la destreza de speaking en el idioma inglés mediante la técnica "Basic Context English" en estudiantes de 5 a 6 años. ConcienciaDigital, 3(2.2), 97-110. https://doi.org/10.33262/concienciadigital.v3i2.2.1248

\section{\Ciencia}

El artículo que se publica es de exclusiva responsabilidad de los autores y no necesariamente reflejan el pensamiento de la Revista Conciencia Digital.

El artículo queda en propiedad de la revista y, por tanto, su publicación parcial y/o total en otro medio tiene que ser autorizado por el director de la Revista Conciencia Digital.
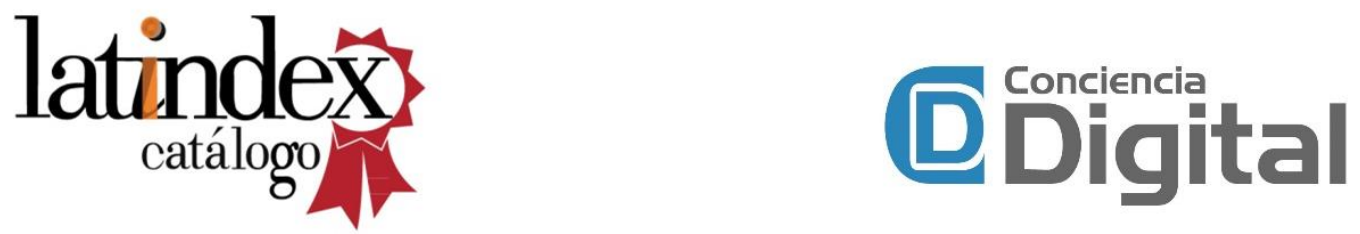